\title{
When is it safe to return to driving following first-ever seizure?
}

\author{
J W L Brown, ${ }^{1,2}$ N D Lawn, ${ }^{1}$ J Lee, ${ }^{1}$ J W Dunne ${ }^{1}$
}

- Additional material is published online only. To view please visit the journal online (http://dx.doi.org/10.1136/ jnnp-2013-307529).

${ }^{1}$ Department of Neurology, Royal Perth Hospital, Perth, Western Australia, Australia ${ }^{2}$ Department of Clinical Neurosciences, University of Cambridge, Cambridge, UK

\section{Correspondence to}

Dr Nicholas Lawn,

Department of Neurology,

Royal Perth Hospital,

GPO Box X2213, Perth,

WA 6001, Australia;

nicholas.lawn@health.wa.gov.

Presented in part at the Epilepsy Society of Australia Annual Scientific Meeting, Perth, Australia 2009 and the Association of British Neurologists Annual Meeting, Bournemouth, England 2010.

Received 23 December 2013 Revised 15 March 2014

Accepted 3 April 2014 Published Online First 25 April 2014

\section{ABSTRACT}

Objectives The risk of recurrence following a first-ever seizure is $40-50 \%$, warranting driving restriction during the early period of highest risk. This restriction must be balanced against the occupational, educational and social limitations that result from patients being ineligible to drive. The recommended duration of non-driving after a first seizure varies widely between jurisdictions, influenced by various factors including the community perception of an acceptable relative level of risk for an accident (the accident risk ratio; ARR). Driving restrictions may be based on individualised risk assessments or across-the-board guidelines, but these approaches both require accurate data on the risk of seizure recurrence.

Methods 1386 patients with first-ever seizure were prospectively analysed. Seizure recurrence was evaluated using survival analysis. The duration of non-driving required for a range of risks of seizure recurrence and ARRs was calculated. Additionally, the actual occurrence of seizures while driving was prospectively determined during follow-up.

Results For a risk of seizure recurrence to fall to $2.5 \%$ per month, corresponding to a monthly risk of a seizure while driving of 1.04 per thousand and an ARR of 2.6, non-driving periods of 8 months are required for unprovoked first-ever seizure, and 5 months for provoked first-ever seizure. Of patients with a seizure recurrence, $14(2 \%)$ occurred while driving, with the monthly risk falling to less than $1 / 1000$ after 6 months.

Conclusions Our data provide a quantitative approach to decisions regarding a return to driving in patients with first-ever provoked or unprovoked seizure.

\section{INTRODUCTION}

Up to $5 \%$ of people will experience a seizure during their lives, many of whom are otherwise healthy adults who work and drive. ${ }^{1}$ The overall risk of recurrence following a first-ever seizure is approximately $50 \%,{ }^{2}$ but this varies with time, markedly decreasing with increasing duration of seizure freedom. Driving restriction is warranted during the early period of highest risk. The period of driving ineligibility varies widely between countries, states and provinces. ${ }^{3}{ }^{4}$ Many states in the USA do not specify durations of driving restriction following first seizure, instead endorsing individual evaluation. After an unprovoked first seizure in Australia, the UK and many European countries, an across-the-board driving ban of at least 6 months is recommended for private vehicle licence holders (cars and motorcycles). ${ }^{356}$ After a provoked first seizure in Australia, a 6-month period of non-driving is also recommended, whereas in the UK, assessment on an individual basis is suggested..$^{5}$ The primary reason for driving restriction is to reduce the risk of a seizure-related accident, but data guiding the duration of driving restriction is sparse. The European recommendation for unprovoked first seizure has recently been supported by subgroup analysis of the multicentre study of early epilepsy and single seizures (MESS). ${ }^{7}$ The outcome of interest in the MESS driving paper was the risk of recurrence over the next 12 months having been seizure-free for 6 months and specifically, as suggested by the UK's Driver and Vehicle Licensing Agency (DVLA), whether this annual risk fell below 20\%. ${ }^{5}$ This analysis assumed a uniform rate of seizure recurrence over 12 months rather than accounting for prominent timedependent changes in the conditional probability of recurrence within that period. The possibility that a driving restriction for less than 6 months may be appropriate for some patients was not explored.

The likelihood of a seizure occurring while driving can be estimated by multiplying the risk of seizure recurrence by the proportion of time spent driving. Additionally, a formula (see online supplementary data) can derive a patient's accident risk ratio (ARR), their relative risk of a motor vehicle accident compared with the general population; and this ARR may be compared with other risk factors for motor vehicle accidents, for example, male drivers aged less than 25 years $(\mathrm{ARR}=7)$, or driving when aged over 70 years (ARR 2). ${ }^{8} 9$ The European Working Group on Epilepsy and Driving concluded that for a first seizure, an ARR of between 2.0 and 3.0 was appropriate. ${ }^{8}$ Once licensing authorities have determined the acceptable ARR, then the formula can be further used to derive the acceptable chance of seizure recurrence for a given time period (see online supplementary data), often expressed as the Chance of Occurrence of a Seizure in the next Year (COSY). We calculated the durations of driving restriction required for monthly rates of seizure recurrence to fall to various levels, with their corresponding ARRs, in an unselected group of patients prospectively studied with either first-ever provoked or unprovoked seizure. We then compared these calculations to the actual rate of seizures while driving.

\section{METHODS}

The Western Australian first seizure database was established in 2000. This is an ongoing prospective study of patients with first-ever seizure seen by a hospital-based epilepsy service aiming to identify outcomes and risk factors for seizure recurrence. 
The methodology has been described previously. ${ }^{10}{ }^{11}$ All patients seen between 2000 and 2011 with first-ever seizure, provoked or unprovoked, were included except those ineligible to drive because of age (<16 years) or disability. Provoked first seizures were those that occurred in the setting of a clearly identifiable proximate cause ${ }^{12}$ and were subdivided into two groups: first, those occurring within 7 days of an acute central nervous system (CNS) insult (eg, head injury, stroke or CNS infection), or with an acute systemic insult and an epileptogenic lesion on CT or MRI, (collectively termed 'CNS lesion'); and second, those related to an acute systemic, metabolic or toxic insult including alcohol and drug withdrawal without an epileptogenic lesion on neuroimaging (termed 'systemic'). Unprovoked first seizures were defined as 'remote symptomatic' if there was history of or neuroimaging evidence for a prior CNS insult (including brain tumors), and 'idiopathic' if there was no obvious cause (encompassing genetic epilepsy syndromes and cryptogenic). Seizure type and electroclinical syndrome (partial, generalised or unclassified) were categorised for the presenting seizure according to published guidelines. ${ }^{12}$ EEG and neuroimaging (CT brain, MRI brain or both) were performed in $97 \%$ and $98 \%$ of patients, respectively. The first EEG after the presenting seizure, a routine, non-sleep-deprived recording, was analysed and classified as being normal or abnormal with abnormalities defined as epileptiform or non-specific. CT and MRI scans were performed according to previously described standardised protocols. ${ }^{13}$ Neuroimaging abnormalities were defined as epileptogenic if a lesion likely relevant to the cause of the seizure was found.

\section{Follow-up}

Patients were reviewed at clinic 3-9 months after the index seizure, and if there had been no further seizures were thereafter contacted by phone every 1-2 years until seizure recurrence occurred or death. For those not contactable by phone the integrated hospital computer system was checked for attendance at other major hospitals in Western Australia, and if so, the related medical records were obtained. Any seizure (provoked or unprovoked) occurring during follow-up was considered a recurrence. If a patient was treated after a first seizure for at least 4 weeks, but subsequently discontinued treatment during follow-up, they were still considered treated. Patients who had a second seizure after being referred but prior to being seen at the clinic were included, with the initial seizure being analysed as the first-ever seizure and the second defined as a recurrence. Patients identified during follow-up (but prior to any recurrence) to have developed a new process after the first-ever seizure that may have contributed to risk of further seizures (eg, stroke, significant head injury) were excluded.

\section{Outcomes and statistical analysis}

The primary outcome was occurrence of a second seizure. The cumulative and conditional (given survival up to a specific time) rates of recurrence were analysed for patients with unprovoked and provoked seizures. Time to seizure recurrence was analysed using Kaplan-Meier curves and log-rank statistics. Conditional probabilities of continued seizure freedom over various time intervals were calculated by dividing the cumulative probability of seizure-freedom at the end of the time interval by that at the beginning of the time interval. ${ }^{14}$

Potential risk factors for seizure recurrence identified in prior first seizure studies ${ }^{2} 111516$ were examined using Cox proportional hazards models. Independent variables analysed were age at first seizure, gender, family history of epilepsy, occurrence of the first seizure as a cluster or status epilepticus, seizure type, timing of seizure (awake vs asleep), seizure aetiology (including epileptogenic lesion on imaging), treatment and epileptiform abnormalities on EEG. Variables significant at the 10\% level in preliminary univariate analyses were included in the multivariate Cox proportional hazards regression models. HR, and 95\% CIs for multivariate analyses were calculated from the final models. Results were considered statistically significant at the 5\% level.

We calculated the time from the first seizure when the monthly conditional risk of seizure recurrence consistently fell (for at least two consecutive months) to 1.7, 2.5 and 3.1\% per month, equivalent to COSYs of 20, 30 and $37 \%$, and the commonly accepted ARRs of 2.0, 2.6 and 3.0 respectively $^{8}$; with a COSY of $30 \%$ and an ARR of 2.6 being in the middle of this accepted range. We have used monthly recurrence because annual risk does not account for the marked time-varying changes in the likelihood of first seizure recurrence within a 1 -year period. We also calculated the equivalent monthly risk of a seizure while driving by multiplying the monthly risk of seizure recurrence by the proportion of time spent driving. This calculation uses an average driving time of $1 \mathrm{~h} /$ day, and assumes that a recurrent seizure has an equal chance of occurring at any time within a $24 \mathrm{~h}$ period, a reasonable assumption after first seizure (and in most patients with established epilepsy).

Additionally, whether the first-ever seizure and any recurrence actually occurred while driving was prospectively ascertained at the initial interview and during follow-up. Actuarial analysis was used to estimate the risk of a second seizure while driving, censoring patients who were not driving at the time of seizure recurrence, or did not have a further seizure.

The first seizure study was approved by the Royal Perth Hospital ethics committee.

\section{RESULTS}

Of 1443 first-ever seizure patients, 1386 who were eligible to drive were studied. Thirty-two patients $(2.2 \%)$ were excluded because of disability, and 25 patients $(1.7 \%)$ were aged less than 16 years at the time of the first seizure. Nine hundred and eighty-eight patients (71\%) had an unprovoked first seizure, and 398 patients $(29 \%)$ had a provoked first seizure. The median time to be seen from the first seizure was 31 days. Median duration of follow-up was 1250 days (range: 4-4389 days). Follow-up by clinic review and/or phone was obtained in $90 \%$ of the patients. In the remaining $10 \%$ information was obtained via the interhospital computer system and medical records. There was no difference in the rate of seizure recurrence between those contacted directly and those followed-up using computer records. Sixty-one patients $(4.4 \%)$ had less than 1 year of follow-up, and at the time of last follow-up had no seizure recurrence, including 15 who died unrelated to seizures. The clinical and investigation findings are summarised in table 1 .

\section{Recurrence after first seizure}

The cumulative probabilities of seizure recurrence for provoked and unprovoked seizures are shown in figure 1 and table 1 . Unprovoked idiopathic and remote symptomatic subgroups have different survival curves (logrank $\mathrm{p}<0.001$ ), as do provoked CNS-lesion and systemic subgroups (logrank $p=0.007)$. The overall likelihood of seizure recurrence at 1 year was $43 \%(95 \%$ CI $40 \%$ to $45 \%$ ): $48 \%$ (95\% CI $45 \%$ to $51 \%$ ) for unprovoked first seizure, and 29\% (95\% CI 24\% to 33\%) for provoked first seizure $(\mathrm{p}<0.0001)$. The time at which conditional probabilities (the monthly risk) of seizure recurrence consistently fell to 1.7, 2.5 and $3.1 \%$ per month (equivalent COSYs of 20, 30 and 
Table 1 Clinical, EEG and neuroimaging findings

\begin{tabular}{|c|c|c|c|c|}
\hline & \multicolumn{2}{|l|}{ Unprovoked } & \multicolumn{2}{|l|}{ Provoked } \\
\hline & $\begin{array}{l}\text { Idiopathic } \\
n=673\end{array}$ & $\begin{array}{l}\text { Remote symptomatic } \\
n=315\end{array}$ & $\begin{array}{l}\text { CNS lesion } \\
n=93\end{array}$ & $\begin{array}{l}\text { Systemic } \\
n=305\end{array}$ \\
\hline Gender male, $n(\%)$ & $410(61)$ & $211(67)$ & $58(62)$ & $170(56)$ \\
\hline Median age at first seizure (range), years & $35(16-88)$ & $45(16-91)$ & $40(16-80)$ & $30(16-85)$ \\
\hline \multicolumn{5}{|l|}{ Seizure type } \\
\hline Tonic-clonic seizure $\mathrm{n}(\%)$ & $647(96)$ & $275(87)$ & $83(89)$ & 304 (99) \\
\hline Simple or complex partial n (\%) & $26(4)$ & $40(13)$ & $10(11)$ & $1(1)$ \\
\hline Family history of seizures (1st degree) $n(\%)$ & $82(12)$ & $19(6)$ & $5(6)$ & $33(11)$ \\
\hline Status epilepticus n (\%) & $5(1)$ & $11(4)$ & $10(11)$ & $4(1)$ \\
\hline Cluster $\mathrm{n}(\%)$ & $110(16)$ & $67(21)$ & $38(41)$ & $62(20)$ \\
\hline Occurrence from sleep n (\%) & $171(26)$ & $62(20)$ & $10(11)$ & $23(8)$ \\
\hline EEG $n(\%)$ & $668(99)$ & $292(93)$ & $87(94)$ & $285(93)$ \\
\hline Epileptiform EEG n (\%) & $104(15)$ & $59(20)$ & $12(14)$ & $34(12)$ \\
\hline Generalised n (\%) & $68(10)$ & $5(2)$ & $0(0)$ & $29(10)$ \\
\hline Focal n (\%) & $36(5)$ & $54(18)$ & $12(14)$ & $5(2)$ \\
\hline \multicolumn{5}{|l|}{ Non-specific abnormality } \\
\hline Generalised n (\%) & $121(18)$ & $77(26)$ & $28(32)$ & $55(19)$ \\
\hline Focal $n(\%)$ & $109(16)$ & $166(57)$ & $35(40)$ & $25(9)$ \\
\hline Treated n (\%) & $105(16)$ & $148(47)$ & $57(62)$ & $32(10)$ \\
\hline Median duration follow-up, days & 1265 & 1381 & 1019 & 1198 \\
\hline Median time to second seizure, days & 109 & 85 & 32 & 119 \\
\hline \multicolumn{5}{|c|}{ Cumulative probability of seizure recurrence $\%(95 \% \mathrm{CI})$} \\
\hline 1 year & 44 (40 to 47 ) & 56 (51 to 62$)$ & 38 (28 to 48$)$ & 26 (21 to 31$)$ \\
\hline 2 years & 50 (46 to 54$)$ & 63 (58 to 68$)$ & 43 (32 to 53$)$ & 29 (23 to 34$)$ \\
\hline 5 years & 58 (54 to 62$)$ & 70 (65 to 76$)$ & 45 (34 to 56$)$ & 32 (27 to 38 ) \\
\hline \multicolumn{5}{|l|}{ Seizure while driving } \\
\hline With first-ever seizure $\mathrm{n}(\%)$ & $31(5)$ & $15(5)$ & $3(3)$ & $6(2)$ \\
\hline With second seizure $\mathrm{n}(\%)$ & $7 / 367(2)$ & $2 / 210(1)$ & $0 / 38(0)$ & $5 / 87(6)$ \\
\hline
\end{tabular}

37\%) with corresponding ARRs are shown in table 2. If the MESS driving study methodology is used, ${ }^{7}$ for patients remaining seizure-free at 6 months, the overall risk of recurrence over the next 12 months (COSY) was $17.2 \%$ (95\% CI $14.6 \%$ to 19.7\%): for unprovoked first seizure $20.4 \%$ (95\% CI $17.1 \%$ to $23.8 \%$ ), and provoked first seizure $10.2 \%$ (95\% CI $6.6 \%$ to $13.8 \%$ ), within the acceptable range as defined by the DVLA for most patients. However, for these same patients who remained seizure-free at 6 months, the overall conditional probability of

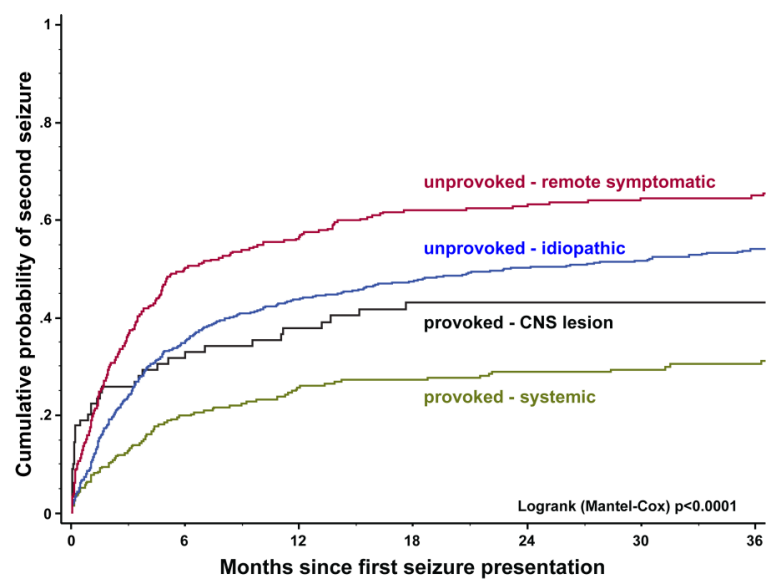

Figure 1 Seizure recurrence according to aetiological subgroup of first seizure. recurrence for the 6-8-month interval was 5\% (95\% CI 3.5\% to $6.5 \%$ ), equivalent to an unacceptably high COSY of $30 \%$, falling for the $16-18$-month interval to $1.4 \%$ (95\% CI $0.5 \%$ to $2.3 \%$ ), equivalent COSY of $8.4 \%$; for unprovoked first seizure,

Table 2 Duration of non-driving required for a private motor vehicle following first seizure (subdivided according to aetiology) using varying acceptable risks of seizure recurrence

\begin{tabular}{|c|c|c|c|}
\hline & \multicolumn{3}{|c|}{$\begin{array}{l}\text { Time needed off the road (months) for } \\
\text { conditional rate of seizure recurrence to fall } \\
\text { to: }\end{array}$} \\
\hline & $\begin{array}{l}1.7 \% \text { per } \\
\text { month }\end{array}$ & $\begin{array}{l}2.5 \% \text { per } \\
\text { month }\end{array}$ & $\begin{array}{l}3.1 \% \text { per } \\
\text { month }\end{array}$ \\
\hline $\begin{array}{l}\text { Equivalent monthly risk of a } \\
\text { seizure while driving* }\end{array}$ & $\begin{array}{l}0.69 \text { per } 1000 \\
(1 \text { in } 1442)\end{array}$ & $\begin{array}{l}1.04 \text { per } 1000 \\
(1 \text { in } 964)\end{array}$ & $\begin{array}{l}1.3 \text { per } 1000 \\
(1 \text { in } 771)\end{array}$ \\
\hline Equivalent ARR† & ARR 2.0 & ARR 2.6 & ARR 3.0 \\
\hline All patients (months) & 8 & 7 & 5 \\
\hline Unprovoked all (months) & 13 & 8 & 7 \\
\hline Remote symptomatic & 17 & 10 & 7 \\
\hline Idiopathic & 12 & 8 & 7 \\
\hline Provoked (months) & 6 & 5 & 4 \\
\hline
\end{tabular}


the risk over the 6-8-month interval was $6.3 \%$ (95\% CI $4.3 \%$ to $8.3 \%$ ), equivalent COSY of $38 \%$, and falling for the $16-18$-month interval to $1.8 \%$ (95\% CI $0.5 \%$ to $3.1 \%$ ).

\section{Seizures while driving}

Fifty-five patients $(4 \%)$ were driving at the time of their firstever seizure (table 1). Fourteen patients (2\%) were driving at the time of the recurrence, seven within 6 months of the first seizure. The overall conditional probability of a second seizure occurring while driving was consistently less than $1 / 1000$ per month after 6 months. Numbers were insufficient for subgroup analysis.

\section{Predictors of seizure recurrence and role of investigations Unprovoked seizure}

Independent predictors of seizure recurrence were remote symptomatic aetiology (HR 1.35, 95\% CI 1.13 to $1.61 ; \mathrm{p}<0.001$ ), partial seizures (HR 1.69, 95\% CI 1.22 to 2.34 ; $\mathrm{p}=0.002$ ), epileptiform abnormality on EEG (HR 1.37, 95\% CI 1.11 to 1.69 ; $\mathrm{p}=0.003$ ), and first seizure from sleep (HR 1.28, 95\% CI 1.06 to $1.54 ; \mathrm{p}=0.01)$. Antiepileptic drug treatment after the first seizure was associated with a higher seizure recurrence rate on univariate analysis, but was not a significant covariate on multivariate analysis or subgroup analysis of remote symptomatic or idiopathic unprovoked first-seizure patients. An epileptogenic lesion on neuroimaging was highly correlated with remote symptomatic aetiology, and was only an independent predictor of recurrence when remote symptomatic aetiology was removed from the multivariate model. While abnormal EEG and/or neuroimaging increased the risk of seizure recurrence somewhat, patients with an unprovoked first seizure who had no neuroimaging or EEG abnormality still had a cumulative probability of seizure recurrence of $23 \%$ (95\% CI $19 \%$ to $26 \%$ ) at 3 months, $34 \%(95 \%$ CI $30 \%$ to $38 \%)$ at 6 months and $45 \%(95 \%$ CI $41 \%$ to $49 \%$ ) at 1 year, with the conditional risk of seizure recurrence falling to $2.5 \%$ per month after 8 months from the first-ever seizure.

\section{Provoked seizure}

Independent predictors of seizure recurrence were an epileptogenic lesion on neuroimaging (HR 2.42, 95\% CI 1.59 to 3.69; $\mathrm{p}<0.001$ ), partial seizures without generalisation (HR 2.94, $95 \%$ CI 1.30 to $6.67 ; \mathrm{p}=0.01$ ) and epileptiform abnormality on EEG (HR 1.79, 95\% CI 1.12 to 2.86; p=0.01). While patients with a CNS lesion had a higher recurrence rate, those with a provoked first seizure related to a systemic cause and without neuroimaging or EEG abnormality still had cumulative probabilities of seizure recurrence of $14 \%$ (95\% CI $10 \%$ to $19 \%)$ at 3 months, $23 \%(95 \%$ CI $17 \%$ to $28 \%)$ at 6 months and $31 \%(95 \% \mathrm{CI} 25 \%$ to $37 \%)$ at 1 year, with the conditional risk of seizure recurrence falling to $2.5 \%$ per month after 5 months from the first seizure.

\section{DISCUSSION}

While every driver has a risk of having an accident, this is increased after a first-ever seizure because of the chance of seizure recurrence. Appropriate first-seizure management includes defining an adequate seizure-free interval before returning to driving, acknowledging that driving restrictions commonly limit occupational, educational and social opportunities.

Our study is prospective, calculates monthly rates of seizure recurrence with first-ever provoked or unprovoked seizure, and compares these calculations with the actual rate of seizures while driving. Our data reveal that after a first-ever seizure, patients overall require 5-8 months off the road before their conditional probability (monthly risk) of seizure recurrence falls to between $1.7 \%$ and $3.1 \%$ per month, equating to the suggested ARR of 2.0-3.0, and an estimated monthly risk of a seizure behind the wheel of 1 in 770 to 1 in 1440 . These calculations are supported by our findings that the actual occurrence of a second seizure while driving is high within the first 6 months, a time when patients have been advised not to drive, but some have continued to do so. Our observational findings on the actual risk of a second seizure while driving are probably an underestimate, given at least some patients would have followed advice not to drive during this time and we rely on patients reporting the circumstances of seizure recurrence accurately. Our data do not support a liberalising of driving restriction after first-ever seizure and suggest that at least a 6-month duration of non-driving is appropriate. Subgroup analysis demonstrates that a driving ban of 8 months is required for unprovoked seizures and 5 months for provoked seizures, including for those without predictors of recurrence such as imaging or EEG abnormalities. A return to driving after 6 months of seizure freedom for patients with unprovoked seizures, as recommended by many jurisdictions, requires that the community accepts an ARR of 3.6, and a return to driving after 3 months as recommended in some US states, ${ }^{3}$ requires that the community accepts an ARR of 6.2.

Our data shows somewhat higher seizure recurrence rates compared to other studies. ${ }^{17-23}$ This likely reflects a number of factors: the hospital-based setting; wider inclusion criteria, enrolment of all patients presenting with first-ever seizure, including a greater proportion of patients with a higher risk of seizure recurrence; enrolment of patients who had a second seizure while awaiting assessment; and defining any second seizure, provoked or unprovoked, as a recurrence. Our findings in patients with provoked seizures are similar to a hospital-based study where any second seizure was also considered a recurrence. ${ }^{24}$ We included a provoked second seizure as a recurrence because the potential adverse sequelae while driving are the same. Most patients (90\%) were referred from hospital emergency departments, or while inpatients under the care of general medicine or other specialties, and most presented with a tonic clonic seizure. In Western Australia, acute care is predominantly provided by public hospital-funded emergency departments, so a high proportion of first-ever tonic clonic seizures are referred to the first seizure service.

Our calculations are based on the assumption that patients are driving for no more than $1 \mathrm{~h} /$ day. Doubling the driving time doubles the risk of a seizure behind the wheel. While a driving time of $1 \mathrm{~h} /$ day may be a reasonable assumption in Europe, ${ }^{8}$ in other countries the average daily driving time is longer: in Australia, the mean daily driving time is $90 \mathrm{~min},{ }^{25}$ so if an ARR of $\leq 2.6$ is accepted, 13 months of non-driving is required for patients with unprovoked first seizures, and 6 months for provoked first seizures.

In the UK and Europe, it has been suggested that provoked seizures be assessed on an individual basis. ${ }^{5} 8$ However, even for patients with a provoked first seizure related to a systemic cause and without neuroimaging or EEG abnormality, the conditional risk of seizure recurrence fell to $2.5 \%$ per month only after 5 months. Our data supports an across-the-board driving ban of at least 5 months for provoked seizures. This approach also avoids the potential difficulties of defining a provoked seizure. $^{26-28}$

Our findings provide a framework to guide decisions regarding the duration of non-driving following first-ever seizure. 
Prospective data documenting the actual rate of seizure-related motor vehicle accidents are needed to validate any recommendations $^{29}$ acknowledging many potentially confounding factors exist, such as variable compliance with driving restrictions, under-reporting of seizures, especially when driving, and underreporting of motor vehicle accidents. ${ }^{30}$

Contributors JWLB: study concept and design, acquisition of data, analysis and interpretation, drafted and revised manuscript, study supervision; NDL: study concept and design, acquisition of data, analysis and interpretation, drafted and revised manuscript, study supervision; JL: acquisition of data, analysis and interpretation; JWD: study concept and design, acquisition of data, analysis and interpretation, drafted and revised manuscript, statistical analyses.

Funding Funding for the establishment and maintenance of the first-seizure database in the form of a grant to cover the costs of a research assistant was received from the Medical Research Foundation of Royal Perth Hospital and UCB-Pharma. Neither source of funding was involved in any way in the design and conduct of the study, collection, management, analysis and interpretation of the data and preparation, review, approval or decision to submit the manuscript.

\section{Competing interests None.}

Ethics approval Royal Perth Hospital Ethics committee.

Provenance and peer review Not commissioned; externally peer reviewed.

\section{REFERENCES}

1 Kotsopoulos IA, van Merode T, Kessels FG, et al. Systematic review and meta-analysis of incidence studies of epilepsy and unprovoked seizures. Epilepsia 2002;43:1402-9.

2 Berg AT. Risk of recurrence after a first unprovoked seizure. Epilepsia 2008;49(Suppl 1):13-18.

3 Winston GP, Jaiser SR. Western driving regulations for unprovoked first seizures and epilepsy. Seizure 2012;21:371-6.

4 Krauss GL, Ampaw L, Krumholz A. Individual state driving restrictions for people with epilepsy in the US. Neurology 2001;57:1780-5.

5 Driver vehicle licensing agency. At a glance guide to the current medical standards of fitness to drive. Swansea, Wales: DVLA, 2012.

6 Assessing fitness to drive. National transport commission. Sydney, Australia: Austroads, 2012. ISBN 978-1-921991-01-1.

7 Bonnett LJ, Tudur-Smith C, Williamson PR, et al. Risk of recurrence after a first seizure and implications for driving: further analysis of the Multicentre study of early Epilepsy and Single Seizures. BMJ 2010;341:c6477.

8 EWGED. A report of the second European working group on epilepsy and driving an advisory board to the driving license committee of the European Union. Epilepsy and driving in Europe. Final report; 3 April 2005. ec.europa.eu/.../epilepsy_and_ driving_in_europe_final_report_v2_en.pdf. Brussels, 2005.

9 Sonnen AE; The European Working Group. Epilepsy and driving proceedings first European workshop epilepsy and driving licences group 1. IBE, 1995.

10 Kho LK, Lawn ND, Dunne JW, et al. First seizure presentation: do multiple seizures within 24 hours predict recurrence? Neurology 2006;67:1047-9.
11 Lawn ND, Kelly A, Dunne J, et al. First seizure in the older patient: clinical features and prognosis. Epilepsy Res 2013;107:109-14.

12 Commission on Epidemiology and Prognosis, International League Against Epilepsy. Guidelines for epidemiologic studies on epilepsy. Epilepsia 1993;34:592-6.

13 Ho K, Lawn N, Bynevelt M, et al. Neuroimaging of first-ever seizure: contribution of MRI if CT is normal. Neurol Clin Pract 2013;3:398-403.

14 Davis FG, McCarthy BJ, Freels $S$, et al. The conditional probability of survival of patients with primary malignant brain tumors. Surveillance, epidemiology, and end results (SEER) data. Cancer 1999;85:485-91.

15 Berg AT, Shinnar $S$. The risk of seizure recurrence following a first unprovoked seizure: a quantitative review. Neurology 1991;41:965-72.

$16 \mathrm{Kim}$ LG, Johnson TL, Marson AG, et al. Prediction of risk of seizure recurrence after a single seizure and early epilepsy: further results from the MESS trial. Lancet Neurol 2006;5:317-22.

17 Marson AG, Jacoby A, Johnson A, et al. Immediate versus deferred antiepileptic drug treatment for early epilepsy and single seizures: a randomized controlled trial. Lancet 2005;365:2007-13.

18 Annegers JF, Shirts SB, Hauser WA, et al. Risk of recurrence after an initial unprovoked seizure. Epilepsia 1986;27:43-50.

19 Jallon P, Smadja D, Cabre P, et al. EPIMART: Prospective incidence study of epileptic seizures in newly referred patients in a French Caribbean Island (Martinique). Epilepsia 1999;40:1103-9.

20 Jallon P, Loiseau P, Loiseau J; on behalf of Groupe CAROLE. Newly diagnosed unprovoked epileptic seizures: Presentation at diagnosis in CAROLE study. Epilepsia 2001;42:464-75.

21 Adelo C, Andell E, Amark P, et al. Newly diagnosed single unprovoked seizures and epilepsy in Stockholm, Sweden: first report from the Stockholm Incidence Registry of Epilepsy (SIRE). Epilepsia 2009;50:1094-101.

22 Hauser WA, Rich SS, Annegers JF, et al. Seizure recurrence after a 1st unprovoked seizure: An extended follow-up. Neurology 1990;40:1163-70.

23 Hesdorffer DC, Benn EK, Cascino GD, et al. Is a first acute symptomatic seizure epilepsy? Mortality and risk for recurrent seizure. Epilepsia 2009;50:1102-8

24 Leung $\mathrm{H}$, Man $\mathrm{CBL}$, Hui $\mathrm{ACF}$, et al. Prognosticating acute symptomatic seizures using two different seizure outcomes. Epilepsia 2010;51:1570-9.

25 NSW Centre for Road Safety. Road traffic crashes in New South Wales. Statistical statement for the year ended 31 December 2008 ISBN 978-1-921692-62-8 ISSN 0155-2546 RTA/pub.09.554

26 Shorvon SD, Guerrini R. Acute symptomatic seizures-should we retain the term? Epilepsia 2010;51:722-3.

27 Beghi E, Carpio A, Forsgren L, et al. Recommendation for a definition of acute symptomatic seizure. Epilepsia 2010;51:671-5.

28 Shorvon SD, Guerrini R, Andermann D. Introduction to the concept of provoked epilepsy. In: Shorvon SD, Andermann F, Guerrini R, eds. The causes of epilepsy. Common and uncommon causes in adults and children. Cambridge: Cambridge University Press, 2011:625-30.

29 Drazkowski JF, Fisher RS, Sirven Jl, et al. Seizure-related motor vehicle crashes in Arizona before and after reducing the driving restriction from 12 to 3 months. Mayo Clin Proc 2003;78:819-25.

30 Dalrymple J, Appleby J. Cross sectional study of reporting of epileptic seizures to general practitioners. BMJ 2000;320:94-7. 